\title{
Il confronto tra centro e periferia nella storia e nel mondo globale
}

\author{
di Giovanni Gozzini
}

Feb 7, 2021 | Centro/Periferia, Emergenza e (nuova) normalità $|\underline{0}|$

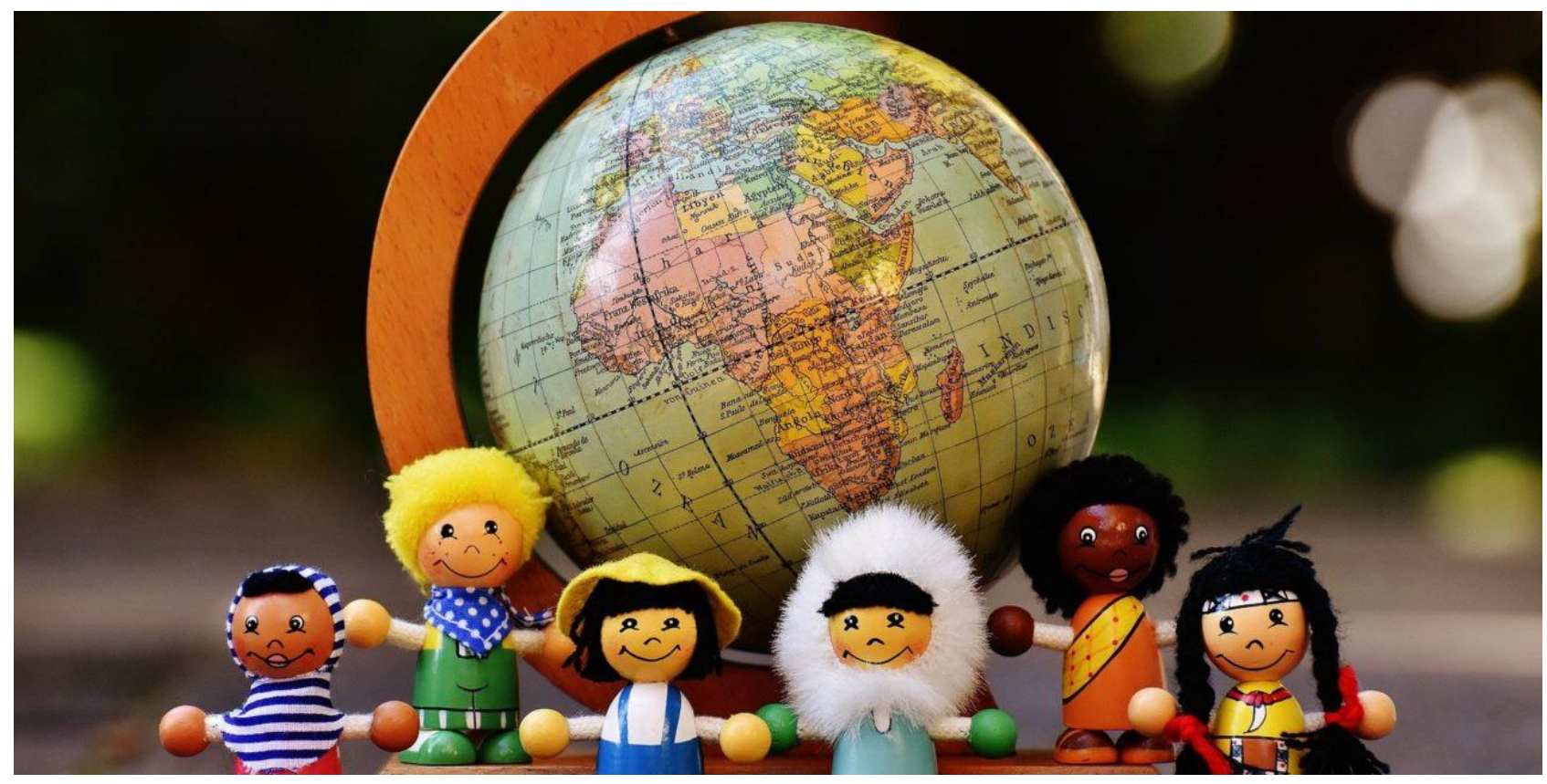

\section{Il dossier sulla Summer school 2020 \\ si compone di 5 coppie di parole chiave. \\ Questo testo afferisce alla coppia di parole chiave \\ Centro/Periferia}

\begin{abstract}
Nella terza giornata della Summer School 2020 abbiamo chiesto a Giovanni Gozzini e Anna Loretoni di riflettere con noi sulle parole chiave Centro e Periferia. Partendo dalla situazione pandemica che ha caratterizzato gli ultimi mesi, Giovanni Gozzini riflette sugli spostamenti di persone e di merci nel mondo globale e globalizzato, ora però limitati dalla diffusione del virus. Attraverso questa chiave di lettura nuova è possibile in realtà analizzare più a fondo - e in una prospettiva storica di lungo periodo - le comuni convinzioni sui concetti di centro e periferia, contrapposti in una statitica solo apparente, che invece è animata da movimenti/mutamenti spesso più fluidi, frequenti e anche caotici di quanto non sembrino.

Centro periferia

In un articolo del 2007 Anthony Fauci elaborò una timeline delle pandemie e delle epidemie influenzali da cui si iniziava a percepire ciò che oggi vediamo realizzarsi nei fatti e che implica un'idea diversa del rapporto centro/periferia. Si pensi ai focolai: guardando ai cluster attivi è evidente che abbiamo bisogno di nuove categorie interpretative per analizzare e ragionare di questi eventi.
\end{abstract}




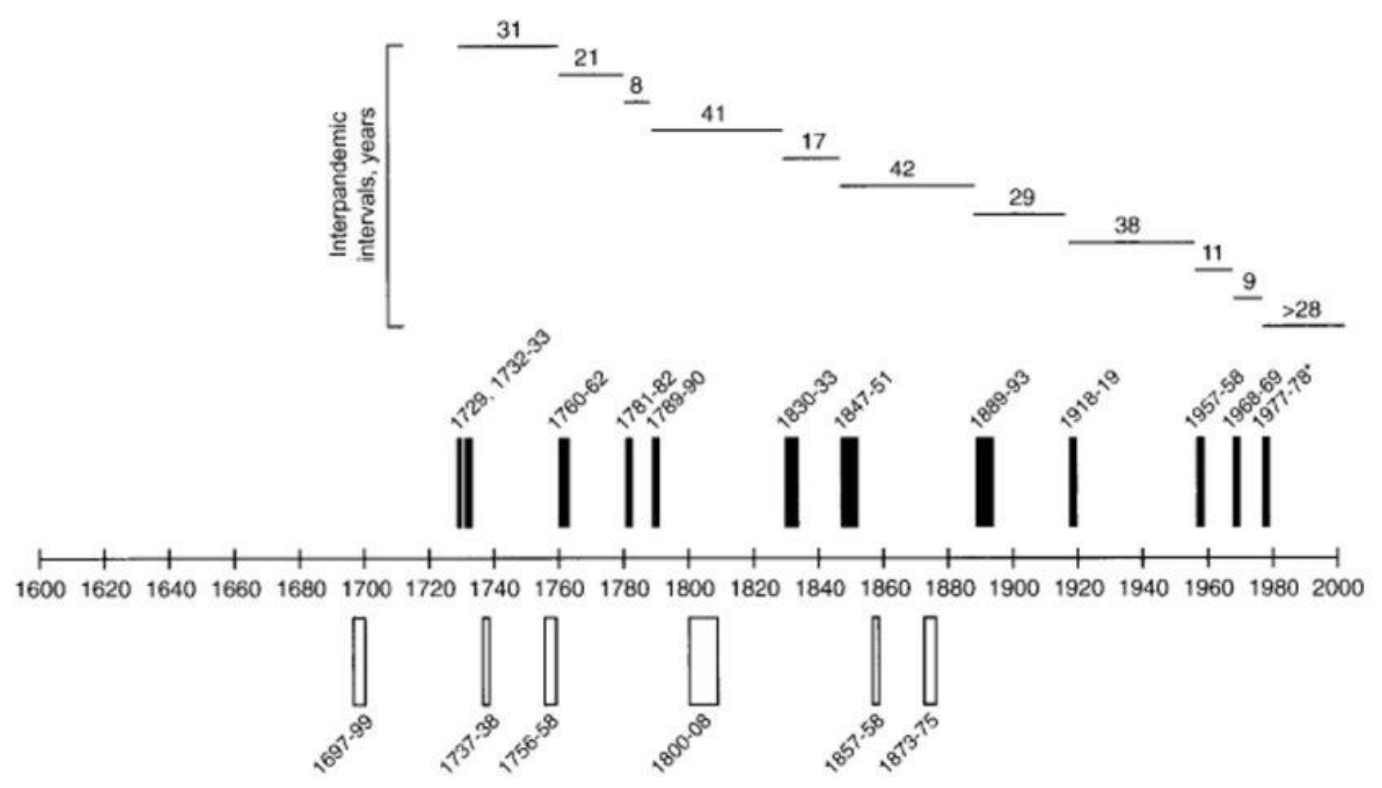

Figura 1. Epidemie di influenza, 1600-2000. Fonte: D.M. Morens-A.S. Fauci, The 1918 Influenza Pandemic: Insights for the 21st Century, «Journal of Infectious Diseases», 195, 2007, 1, Figure 6. Le barre nere sono pandemie, quelle bianche epidemie. Le cifre indicano l'intervallo in anni.

Esiste una regolarità? Secondo l'autore no, ma osservando gli intervalli medi Fauci mostrava come fosse passato troppo tempo dall'ultima pandemia e come bisognasse porre attenzione alla successiva. L'attuale Sars COV2 è stato preceduto nel 2002 dal Sars COV1, primo Corona virus non influenzale della storia che si sviluppò solo in Cina causando 8000 morti, ma con una mortalità più che doppia rispetto a quella attuale.

Cosa ci dicono questi dati? Una cosa consolante: che nonostante la globalizzazione e i movimenti oltre frontiera (1.300.000.000, oggi) le bacchette non hanno accelerato il loro ritmo di ricorrenza nel tempo. La globalizzazione, insomma, non produce una moltiplicazione dei contagi.

La cosa importante però sta nel fatto che queste malattie ci devono portare a cambiare ordine di pensiero. Finora noi abbiamo obbedito a un paradigma diffusionista (c'è un centro, c'è una periferia): esiste un paziente 0 e poi la malattia si diffonde (come nel caso dell'influenza spagnola del biennio 1918-20), si cerca di dimostrare che esiste un centro da cui si origina tutto. Come nella teoria dell'untore: dare la colpa a qualcuno semplifica la realtà e soprattutto ci fornisce un alibi[1].

Secondo Fauci e l'epidemiologia più recente, invece, la diffusione avviene a grappoli, per focolai o cluster simultanei. Tanti centri e tante periferie. 


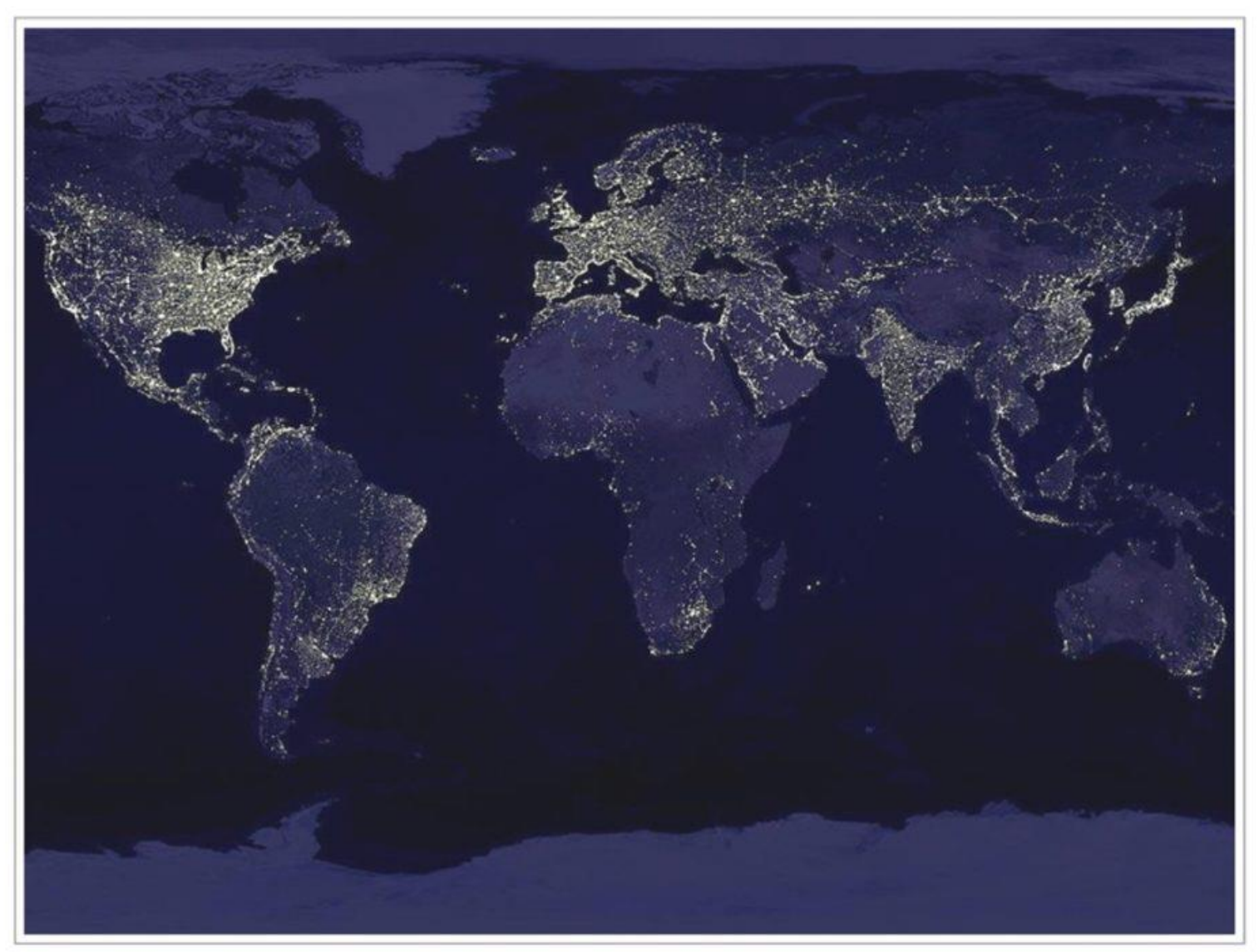

Figura 2

La seconda immagine ci mostra un planisfero nel quale è rappresentata la diffusione della luce artificiale. È un indicatore abbastanza attendibile di due processi storici: l'urbanizzazione e l'industrializzazione. Anche qui: possiamo riconoscere un centro e una periferia? No. Piuttosto molti centri e molte periferie. Se noi guardiamo l'India, l'Europa, il Giappone la diffusione dei due fenomeni è legata a diversi cluster.

Insomma è inutile domandarsi chi sia il paziente zero. Conta molto di più capire come si evolvono le reti.

Basti pensare a quanto sta accadendo con le misure di distanziamento fisico: un inedito dal punto di vista storico. I primi episodi di chiusura delle scuole risalgono al secolo scorso con la già citata epidemia di Spagnola. Ma quella che stiamo vivendo è la prima grande pandemia contrastata contemporaneamente da i governi di tutto il mondo. È la prima volta che l'ingerenza dello Stato impatta così pesantemente nella vita quotidiana delle persone; persino più di quanto accadde durante i totalitarismi e le due guerre mondiali.

Durante i bombardamenti, ad esempio, la gente era costretta a scappare nei rifugi, ma scuole e ristoranti funzionavano. Solo alcuni gruppi ben individuati di discriminati, come gli ebrei, avevano dovuto sopportare reclusioni forzate di un genere paragonabile a quello attuale (oltre a tutto il resto che a noi oggi invece viene risparmiato).

Stiamo senz'altro vivendo un esperimento storico inedito e pieno di implicazioni da studiare. 


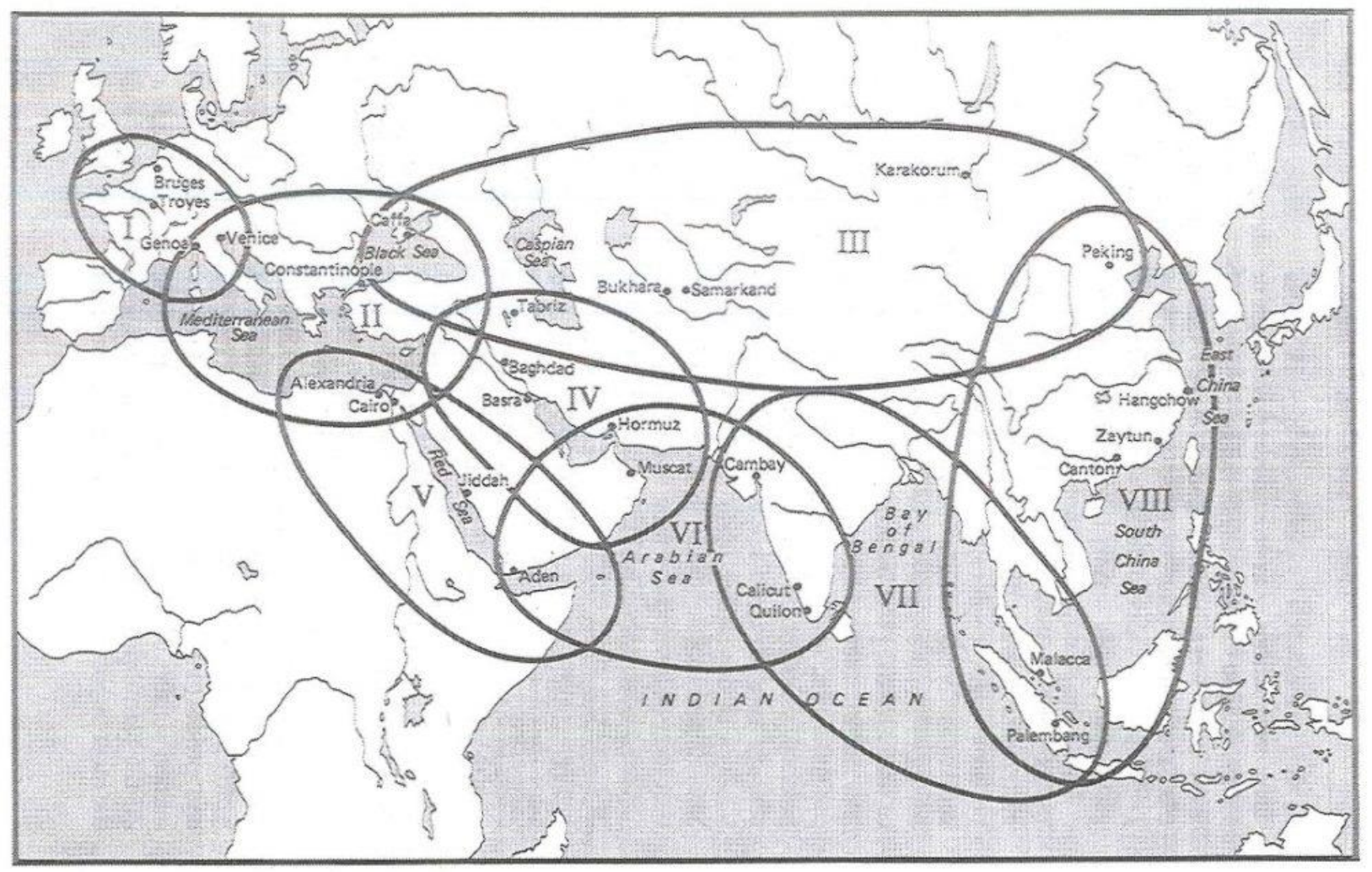

Figura 3

La terza immagine è tratta da Before the European Hegemony: The World System 1250-1350, di Janet Abu Lughod[2] e mette in discussione l'idea stessa di diffusionismo; un sistema categoriale elaborato da Immanuel Wallerstein[3] e secondo il quale dal Quattro-Cinquecento l'economiamondo capitalistica funziona con un centro - diversi succedono l'uno all'altro alla guida:

Repubbliche marinare italiane, Olanda, Gran Bretagna, Stati Uniti; ora forse sarà il tempo della Cina? -, una semiperiferia formata da paesi con cui il commercio del centro entra in relazione di scambio reciproco, e una periferia non toccata dallo sviluppo. Abu Lughod contraddice Wallerstein poichè dimostra l'esistenza di almeno otto bacini commerciali in cui, già ben prima dell'ascesa di Venezia, la gente scambiava merci e monete con viaggi al lunga distanza. Quello di Wallerstein, insomma, è un classico errore causato da un vizio etnocentrico. 


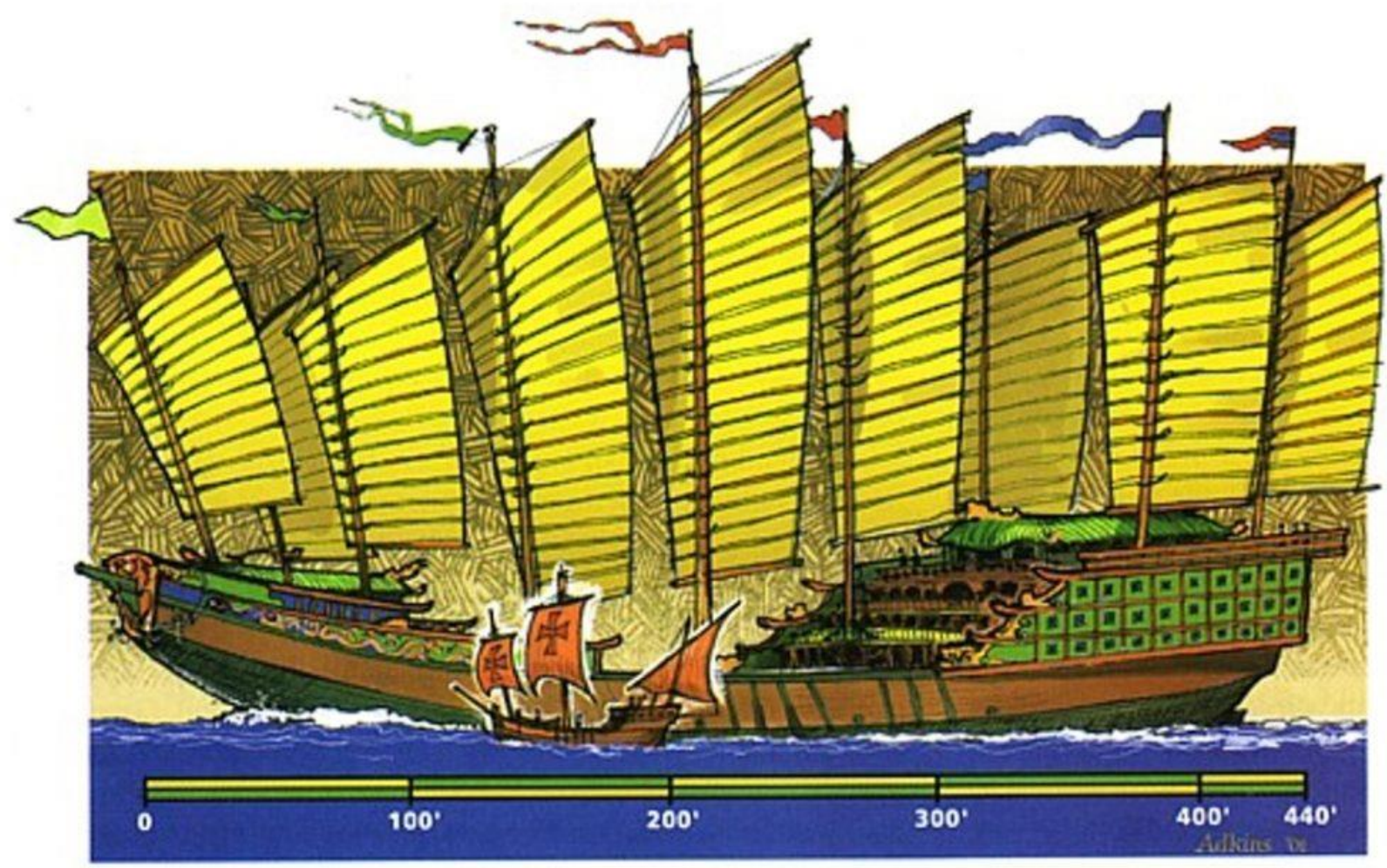

Figura 4

La quarta immagine permette il confronto fra una delle tre caravelle e una del centinaio circa di giunche della flotta imperiale cinese (quelle che fra il 1407 e il 1433 svolsero sette spedizioni, muovendosi dal porto di Canton attraverso l'Oceano indiano fino alle coste del Madagascar e dell'Africa). È una storia ben nota che dimostra l'enorme superiorità - già nel XV secolo - della cantieristica cinese su quella occidentale.

Se quella superiorità lascia il campo ai Colombo e ai Vespucci è solo per una scelta deliberata e consapevole dell'imperatore cinese che scorge il pericolo mortale della formazione di un ceto di mercanti potenzialmente antagonista a quello dei mandarini, i funzionari che gestivano la macchinastato imperiale. Per quattro secoli l'impero cinese consegna all'Europa il dominio dei mari, ma salva se stesso dalla rivoluzione borghese che non risparmierà il Vecchio Mondo europeo. 


\section{Gap tecnologico Cina/Europa, 0-1500 d.C.}

- cromatura dei metalli (17 secoli)

- balestra e trivellazione (13 secoli)

- ponti sospesi e porcellana (12 secoli)

- ghisa, carta e carriola (10 secoli)

- polvere pirica e stampa (5 secoli)

- bussola (2 secoli)

Figura 5

L'ultima immagine è ricavata dagli studi di Joseph Needham e dei suoi allievi[4] e illustra i secoli di ritardo tecnologico dell'Europa rispetto alle innovazioni introdotte in Cina e poi, in epoche diverse, portate fino a noi spesso attraverso la via della seta e le carovane degli arabi.

In Sette brevi lezioni di fisica impariamo da Carlo Rovelli[5] che ancora più importanti degli oggetti, su cui più facilmente appuntiamo la nostra attenzione, sono le relazioni tra di essi. I flussi (di merci, soldi, persone, idee) attraversano i confini molto più di quanto una storia tradizionale fatta di re e di guerre - possa testimoniare. Tale dimensione vale anche per i nostri concetti di centro/periferia. C'è molta più confusione e movimento (e quindi intreccio e contaminazione reciproca, anche per quanto riguarda le malattie) di quanto non si sospetti.

A tutti noi piacerebbe un mondo più ordinato e meno challenging ed è anche per questo motivo che nascono beni-rifugio ideologici piccoli (la Padania o la Catalogna) grandi (islamismo) o medi (razzismo, suprematismo, nazionalismo-populismo).

\section{La globalizzazione a scuola}

I paradigmi per comprendere il mondo sono in rapido cambiamento e sarebbe molto importante che gli insegnanti imparassero ad adottare strategie per portare la complessità della globalizzazione nelle loro classi.

Un modo per farlo è restringere il concetto stesso di globalizzazione e collocarlo nel tempo. Ai docenti il compito di spiegare come la globalizzazione sia fatta di movimenti transfrontalieri di capitali, merci, persone, idee, culture, informazioni, scienza. Seguendo questa logica ci rendiamo conto che gli ultimi due secoli hanno visto succedersi fasi di globalizzazione e de-globalizzazione. 
La prima globalizzazione dell'età contemporanea possiamo farla coincidere con Jules Verne che nel 1873 scrive Il giro del mondo in ottanta giorni. Solo qualche anno dopo, nel 1884, una giornalista, Nellie Bly, racconta per il New York World (il cui leggendario direttore-proprietario è Joseph Pulitzer) il suo personale giro del mondo, compiuto in 73 in giorni.

Passa ancora qualche anno e Picasso lavora su stampe giapponesi e maschere di legno africane per sperimentare il il cubismo. Nel mentre i capitali inglesi arrivano in Argentina a finanziare lo sviluppo delle ferrovie, le locomotive prodotte in Gran Bretagna arrivano in India, i nostri bisnonni vanno nelle Americhe.

La storia, a questo punto, ci insegna che la politica può fermare la globalizzazione, ciò che di solito si fa con la guerra. Arno Mayer[6], ad esempio, interpreta la Prima guerra mondiale come il canto del cigno, l'ultima furibonda reazione dell'aristocrazia contro i mercanti borghesi.

Un altro strumento per fermare la globalizzazione è il protezionismo. Dopo la crisi del 1929 entra in campo e prepara una nuova guerra rompendo la collaborazione tra governi.

Come storici questo dobbiamo dirlo: quando si mettono in campo le barriere doganali contro l'importazione di prodotti di altri stati, si comincia a sentire puzza di bruciato, odore di guerra. È accaduto nel 1914 e dopo il 1929.

Certo per nostra fortuna Trump non è più Presidente degli Stati Uniti d'America, e tuttavia non è detto che la sua stessa tentazione possa venire ad altri capi di stato.

La seconda globalizzazione arriva dopo la Seconda guerra mondiale. Il periodo 1945-1973 ("età dell'oro" secondo Hobsbawm ne Il secolo breve) è l'epoca in cui - fino al primo shock petrolifero il mondo - compreso il continente africano - cresce moltissimo. Processo accompagnato da una forte ripresa degli scambi internazionali. Gli Usa sostituiscono il Regno Unito come fulcri dell'economia mondiale, il dollaro subentra alla sterlina come valuta di scambio a livello globale. Il blocco sovietico si pone alla testa del movimento di decolonizzazione, mentre nuovi colossi (Cina, India) si affacciano sulla scena economica. La fine di questa seconda globalizzazione coincide con le prime preoccupazioni ambientali[7], la crisi dell'impero americano in Vietnam seguita dalla sua resurrezione con Reagan nel 1980.

Parlare, come si fa spesso, di neoliberismo come momento di rottura con il periodo precedente è un po' semplicistico. Basti pensare che al congresso del partito comunista cinese del dicembre $1978-$ solo qualche mese prima della vittoria elettorale di Margaret Thatcher - Deng Xiaoping lancia le "Quattro modernizzazioni", la prima delle quali è rivolta ai contadini. Il leade invita esplicitamente alla «responsabilità familiare», cioè all'arricchimento individuale che può essere perseguito attraverso la liberalizzazione dei prezzi e della produzione agricola. Nei vent'anni successivi 400 milioni di cinesi escono dalla soglia di povertà (misurata dalla Banca Mondiale con la cifra di meno di un dollaro al giorno). Gorbacev qualche anno dopo proverà a fare lo stesso in Unione Sovietica, dove però i contadini, azzerati nelle loro capacità imprenditoriali da decenni di pianificazione, non si trovano nella condizione di reagire.

Solo più tardi, negli anni Novanta, la Cina si apre alla globalizzazione commerciale. Cinque zone le più popolate della costa orientale - sono proclamate duty free: chi viene a produrre qui, ottiene per cinque anni vantaggi fiscali, sindacali e ambientali. Nasce la spinta alla delocalizzazione delle imprese occidentali, di cui continua a fare le spese la nostra forza lavoro meno qualificata. 
Si tratta di una caratteristica inedita rispetto alle globalizzazioni del passato: da sempre i capitali e le merci si sono sempre spostati e delocalizzati a livello globale, ma non le fabbriche. È questo uno dei problemi - gli altri sono: clima, finanza, migrazioni, colossi del web - che la politica, operante ancora solo a livello degli stati-nazione, non riesce ad affrontare. Senza una vera collaborazione internazionale, infatti, nessuno di questi problemi potrà essere risolto. Sperare di farlo, come sostengono i sovranisti, è solo un tentativo di gettare fumo negli occhi.

Cosa è possibile risolvere quando si sostiene che i migranti debbano bussare alla porta accanto? Finché la politica non capirà che le grandi sfide si risolvono collaborando sarà sempre impotente; e continuerà a non governare questi processi.

\section{Ostacolare le migrazioni?}

Se guardiamo la mappa disegnata in Storia e geografia dei geni umani[8] possiamo riflettere sulla scoperta del DNA. Attraverso l'estrazione e l'analisi del DNA contenuto negli scheletri dei nostri antenati preistorici, un ricercatore come Cavalli Sforza ritrova lo stesso ceppo di DNA in tutte le più lontane propaggini secondo lungo una linea rossa. Viene, così, definitivamente archiviata l'ipotesi poligenista, secondo cui diverse razze umane sarebbero apparse più o meno contemporaneamente sulla faccia della terra.

Apparteniamo, invece, tutti alla stessa razza umana, con medesimo DNA: un solo genotipo parte dall'Africa centrale e migra nei vari continenti. Con il tempo il fenotipo cambia per effetto del clima e della luce - ed è proprio il colore della pelle che colpisce e continuerà a colpire la nostra immaginazione - ma il genotipo rimane lo stesso.

Siamo dunque tutti discendenti dallo stesso antenato africano "negro" (per ricorrere a un abusato termine dispregiativo, diventato fuorilegge in un'atmosfera sempre più politicamente corretta).

Eppure a a ben vedere nella mappa di Cavalli Sforza la cosa più interessante sono le frecce che ci mostrano come i nostri antenati preistorici fossero migranti naturaliter: cacciatori e raccoglitori, con nessun mobile al seguito. Una volta prosciugato un ambiente delle sue risorse, essi si spostavano in branco nel successivo.

La migrazione, dunque, sta nel nostro DNA culturale: è nel nostro imprinting originario e quindi nel nostro destino. Ma per noi, dopo secoli di campi coltivati, di città e confini, ha smesso di essere una condizione naturale. Non la riconosciamo più nei nostri simili che ne sono protagonisti. Nemmeno - ed è davvero un paradosso - quando a migrare dal Veneto o dalla Sicilia sono stati nostri bisnonni.

In questa (immagine-figura-grafico...non so cosa sia) vediamo le migrazioni internazionali nel primo tempo della globalizzazione (1840-1940). Anche qui abbiamo abbandonato un punto di vista eurocentrico. Prima consideravamo soltanto la parte relativa all'Atlantico, poi ci siamo accorti che, in seguito all'emancipazione dei servi della gleba in Russia (1861), molti contadini senza terra emigrano in Siberia per sopravvivere. La dimensione delle frecce indica la consistenza numerica degli spostamenti. E poi abbiamo anche i circuiti dell'Oceano indiano: Gandhi nei primi anni del Novecento si sposta dall'India al Sudafrica come avvocato dei diritti civili degli indiani emigrati a lavorare nelle miniere d'oro del Sud Africa: i coolies, di cui si parla nei romanzi di Salgari). 
L'ultima (immagine-figura-grafico...non so cosa sia) ci presenta la migrazione odierna, nel secondo tempo della globalizzazione. Qui possiamo osservare grossomodo quattro poli attrattori verso cui muovono le periferie del mondo: l'Europa, che da terra di emigrazione è diventata terra di immigrazione e gli Stati Uniti, oggi, come un secolo fa, massima calamita per i migranti. Infine due nuovi centri sono rappresentati dai paesi del Golfo Persico (Emirati arabi ricchi di petrodollari e di opere pubbliche ma con poca classe operaia nazionale) e l'Australia.

Una situazione che andrebbe compresa con minore superficialità di chi pensa che i migranti invadano le terre che raggiungono. Come storici e come educatori dovremmo sottolineare come le rimesse - cioè i soldi che i migranti mandano a casa - stanno raggiungendo in cifra assoluta gli investimenti delle multinazionali (ma, a differenza di questi ultimi, vanno direttamente nelle situazioni di bisogno e non nelle casse di imprese già ricche).

I migranti non sono persone che vogliono fare del male o impossessarsi delle cose altrui: si muovono perché vogliono condizioni migliori di vita, in quanto nel loro paese vivono situazioni peggiori delle nostre. I migranti sono attori razionali: la maggior parte di loro risparmiano il più possibile per mandare la cifra più alta possibile a casa. Le nazioni che ne attraggono di più (Stati Uniti e Germania) hanno tassi di disoccupazione inferiori perché i migranti vanno ad occupare posti di lavoro non qualificati e faticosi (servizio domestico, raccolte estive, ecc...) di cui gli indigeni non si occupano più. Il contributo dei migranti al PIL sta diventando sempre più importante e lo sarebbe ancora di più se gli imprenditori che li impiegano accettassero di non farlo al nero per evadere le tasse.

La rivista oggi più importante nel campo della storia e della sociologia delle migrazioni è la francocanadese Diaspora. Siamo abituati a pensare che la diaspora sia qualcosa che riguarda soltanto gli ebrei. Quella particolare esperienza, però, è diventata la chiave interpretativa di tutti i movimenti internazionali di persone, di ogni tempo della storia e di ogni spazio geografico del mondo.

Diaspora vuol dire che il migrante non è una persona che cancella la propria identità di partenza, ma qualcuno che manda i soldi a casa e che forse in quella casa ci vuole tornare. Ne sono un esempio gli studi sulle donne turche che lavorano in Germania. Di solito ci restano dai cinque agli otto anni: non prendono mai la cittadinanza, non rinunciano al velo, non rinnegano le radici religiose perché vogliono tornare a casa. Una volta tornate, però, aprono conflitti con le famiglie e le comunità originarie perché vogliono affermare il loro ruolo di donne: a partire dalle scelte matrimoniali, di vita sessuale e riproduttiva, di gestione dei bilanci domestici. In Germania hanno imparato qualcosa cui non vogliono rinunciare.

Questa modalità non piace agli Stati Uniti che per decenni non hanno tenuto statistiche dei rimpatri in quanto sono affezionati all'idea dell'assimilazionismo: chi va là scopre una nuova vita migliore e non ha più voglia di ritornare a casa (è l'American dream).

Per alcuni ha funzionato e funziona ancora così, ma per altri no. Eppure i migranti di ritorno quando sono stati bene accolti - diventano gli ambasciatori di un dialogo che può cambiare per la prima volta nella storia umana i rapporti tra civiltà e culture. Se i Marco Polo si moltiplicheranno le relazioni tra Cina e resto del mondo sono destinate a cambiare. 
[1] Tesi piuttosto nota che si può trovare in particolare in R. Girard Il capro espiatorio, Adelphi, Milano 1982.

[2] J. Abu Lughod, Before the European Hegemony: The World System 1250-1350, OUP, New York 1989.

[3] (Il sistema mondiale dell'economia moderna, Il Mulino, Bologna 1976-1984.

[4] J. Needham, Science and Civilization in China, Cambridge University Press, Cambridge 19541999.

[5] C. Rovelli, Sette brevi lezioni di fisica, Adelphi, Milano 2014.

[6] A. J. Mayer, Il potere dell'ancien regime fino alla prima guerra mondiale, Laterza, Roma-Bari 1999.

[7] Come emerge per la prima volta in D. H. Meadows, D. L. Meadows; J. Randers; W. W. Behrens III, I limiti dello sviluppo, Mondadori, Milano 1972.

[8] Luca Cavalli Sforza, Storia e geografia dei geni umani, Adephi, Milano 1997. 